\title{
Preliminary Study on The RT-LAMP Assay for Rabbit Hemorrhagic Disease Virus Type 2 Detection
}

\author{
Zexiao YANG* \\ College of Veterinary Medicine \\ Sichuan Agricultural University, \\ Chengdu, China; \\ e-mail: yzxyang2003@126.com
}

\section{Zhengqun MENG}

College of Veterinary Medicine

Sichuan Agricultural University, Chengdu, China;

e-mail: yzxyang2003@126.com

\section{Han ZHANG}

College of Veterinary Medicine Sichuan Agricultural University,

Chengdu, China;

e-mail: yzxyang2003@126.com

\section{Xiaoxue XIANG}

College of Veterinary Medicine

Sichuan Agricultural University,

Chengdu, China;

e-mail: yzxyang2003@126.com

Yadong LIU

College of Veterinary Medicine

Sichuan Agricultural University,

Chengdu, China;

e-mail: yzxyang2003@126.com

\section{Yilin LUO}

College of Veterinary Medicine

Sichuan Agricultural University,

Chengdu, China;

e-mail: yzxyang2003@126.com

\section{Xueping YAO}

College of Veterinary Medicine

Sichuan Agricultural University,

Chengdu, China;

e-mail: yzxyang2003@126.com

\section{Yin WANG}

College of Veterinary Medicine

Sichuan Agricultural University,

Chengdu, China;

e-mail: yaanwangyin@tom.com

Lijun ZHOU

College of Veterinary Medicine

Sichuan Agricultural University,

Chengdu, China;

e-mail: yzxyang2003@126.com

Yan LI

College of Animal Science and Technology

Sichuan Agricultural University,

Chengdu, China;

e-mail: liyan@sicau.edu.cn

\begin{abstract}
To develop a rapid molecular biological method for the detection of rabbit hemorrhagic disease virus type 2 (RHDV2), a set of 4 primers were designed according to the conserved sequence fragment of the capsid protein(VP60) gene of RHDV2 published in GenBank, and the reverse transcriptase loop-mediated isothermal amplification (RT-LAMP) assay was established through identification of target gene fragments, optimization of reaction conditions, sensitivity and specificity tests, and the application tests of 30 samples.The results showed the RT-LAMP method for the RHDV2 detection had a ladder-like pattern of amplification bands from about $119 \mathrm{bp}$ incubation at $63.5^{\circ} \mathrm{C}$ for $90 \mathrm{~min}$ by using agarose gel electrophoresis, the sensitivity of the RT-LAMP could reach about 180 copies/ $\mu \mathrm{L}$ of target fragments, there was no amplification for RHDV, pGM-T-EBHSV, Pasteurella multocida, E.coli and Salmonella from rabbits detection by this approach. The application tests
\end{abstract}

results showed that there were not RHDV2 in the experimentally infected samples and clinical samples. The RT-LAMP assay established here had good specificity and sensitivity, which is suitable for RHDV2 rapid detection.

Keywords-rabbit hemorrhagic disease; RHDV2; RT-LAMP; PCR; detection

\section{INTRODUCTION}

Rabbit hemorrhagic disease (RHD), an important disease in the OIE List, is a highly contagious and rapidly fatal viral disease of both domestic and wild rabbits (Oryctolagus cuniculus) [1]. The disease is caused by rabbit hemorrhagic disease virus (RHDV) and characterized with the typical pathological lesions including necrotic hepatitis, hemorrhaging and edema of lungs and trachea, short 
incubation period (24-48 $\mathrm{h}$ after infection), and high mortality rate of $60-100 \%$ to the adult rabbits [2]. Since RHD was first described in China in 1984, it has been reported in many countries throughout the world successively, and caused significant losses in the rabbit industry [3]. Nowadays, RHDV outbreaks still occur on almost all continents including most parts of Europe, Asia, and parts of Africa, Australia etc. and cause different mortality rates, Although the efficient commercial vaccines protect against RHDV has enabled its control in rabbitries [4, 5].In the summer 2010, a new type rabbit hemorrhagic disease(named RHD2) caused by rabbit hemorrhagic disease virus type 2(RHDV2 or RHDVb)was reported in France both in rabbitries and in wild populations, which generates the similar pathogenic changes with RHD [6].Importantly,RHD2 can affect the rabbit kits under 30 days of age, RHD-vaccinated rabbits and other lagomorphs like different hare species, although RHDV2 had only about $20 \%$ mortality in the animal tests $[6,7]$. Since the first emergence report of RHD2 in France, RHD2 has spread to several European countries and the African continent, such as Italy, Germany, Portugal mainland, England, Wales, several islands of the Azores, etc[8].And there are no the appearances reports about RHD2 cases in China and other countries until now, it is very important and necessary to carry out the researches about the methods for RHDV2 detection to strengthen the inspection and quarantine, along with the development of international economic and trade such as rabbits and its related products introduction.

As a novel nucleic acid amplification method, which was reported in 2000, the RT-LAMP assay has been used for many pathogens detection because of the advantages of rapidity, sensitivity, and non-requirements of any expensive special equipment such as a thermal cycler [9, 10, 11, 12]. In this study a reverse transcriptase loop-mediated isothermal amplification (RT-LAMP) assay was preliminary established and evaluated for RHD2 diagnosis after a serial of tests including identification of target gene fragments, optimization of reaction conditions, sensitivity tests, specificity tests, and the application tests of 30 samples.

\section{MATERIALS AND METHODS}

\section{A. Reagents and Samples.}

TIANprep Mini Plasmid Kit (DP103), 2×Taq PCR MasterMix (KT201) and TIANgel Midi Purification Kit (DP209) were provided by the TIANgen Biotech(Beijing) Co., Ltd, DNA Maker DL2000, EcoRII (1232A), Primerscript RT reagent Kit (BK1901) and RNAiso Plus were provided by the TaKaRa Biotechnology(Dalian) Co., Ltd. Bst 2.0 DNA polymerase (M0537S) was provided by Beijing New England Biolabs Co., Ltd., RHDV-Sch01, VP60 gene fragments of European brown hare syndrome virus (pGM-T- EBHSV), VP60 gene fragments of RHDV2(pMD-19T-RHDV2), Pasteurella multocida, E.coli and Salmonella from rabbits, 5 RHDV experimentally samples infected with the RHDV-Sch01 and 25 clinical samples(stored at $-70{ }^{\circ} \mathrm{C}$ ) were provided by Sichuan Agricultural University animal quarantine Lab.

\section{B. Primer Preparation.}

According to the VP60 gene sequences of RHDV and RHDV2 published in GenBank, 2 specific primers (P1/P2) for RHDV RT-PCR, 2 specific primers (P3/P4) for RHDV2 RT-PCR and 4 LAMP primers for RHDV2 detection (listed in Table I) were carefully designed using DNAStar and Primer Explorer V4 software (http://primerexplorer.jp/e/) subsequently. All of these primers were prepared by the BGI Sequencing Co., Ltd.

TABLE I. PRIMERS USED FOR RHDV2 RT-LAMP STUdy

\begin{tabular}{|c|c|c|c|}
\hline $\begin{array}{c}\text { Task and } \\
\text { Primer name }\end{array}$ & Sequence $\left(5^{\prime}-3^{\prime}\right)$ & $\begin{array}{l}\text { Products } \\
\text { Size(bp) }\end{array}$ & $\begin{array}{c}\text { Reference } \\
\text { sequence }\end{array}$ \\
\hline $\begin{array}{l}\text { RHDV2 RT- PCR } \\
\text { P1 }\end{array}$ & CATATCCACCCCAAACAGT (1275-1293) & 435 & KP129339 \\
\hline $\mathrm{P} 2$ & CCCAAGTTGTACACAAGC (1709-1692) & & \\
\hline RHDV2 RT-LAMP & & $>119$ & KP129339 \\
\hline $\mathrm{R} 2 \mathrm{~F} 3$ & TCCACCCCAAAYAGTRRTGC (1279-1298) & & \\
\hline $\mathrm{R} 2 \mathrm{~B} 3$ & CAGTGGTTGTGAYCCCGC (1476-1459) & & \\
\hline R2FIP & CAAYRGGAGCGGCAGCAGGGAGGATTGTYAATGCACCTGG & & \\
\hline R2BIP & CACCCATYATGTTYGCGTCTGTTTAGYTGAACCGGCCTCA & & \\
\hline \multicolumn{4}{|l|}{ RHDV RT-PCR } \\
\hline P3 & GGGTGTCATATCCACCCCAAA (1269-1289) & 441 & DQ205345 \\
\hline P4 & CCCAGGTTGAACACGAG (1709-1693) & & \\
\hline
\end{tabular}




\section{Identification of RHDV2 Target VP60 Gene Fragment}

To identify the conserved target VP60 gene fragment of RHDV2 was synthesized in vitro using an overlap extension PCR, the constructed recombinant plasmids pMD-19T-RHDV2 was identified by were subsequently identified by PCR (using P1/P2 and MR13-47/RV-M as primers separately) and by sequencing analysis as described in[13].

The PCR reaction volume $(50 \mu \mathrm{L})$ containing $25 \mu \mathrm{L}$ of $2 \times$ Taq PCR MasterMix, $1 \mu \mathrm{L}(10 \mu \mathrm{mol} / \mathrm{L})$ of each of the primers (P1/P2 and MR13-47/RV-M), $5 \mu \mathrm{L}$ of the last secondary extension reaction products and $18 \mu \mathrm{L}$ ddH2O. The PCR procedure program were: denaturing at $95{ }^{\circ} \mathrm{C}$ for 5 min, followed by 35 cycles at $94{ }^{\circ} \mathrm{C}$ for $40 \mathrm{~s}, 55{ }^{\circ} \mathrm{C}$ for $30 \mathrm{~s}$, and $72{ }^{\circ} \mathrm{C}$ for $30 \mathrm{~s}$, then terminated by an elongation at $72{ }^{\circ} \mathrm{C}$ for $10 \mathrm{~min}$. In all, $5 \mu \mathrm{L}$ of PCR products were analyzed using $10 \mathrm{~g} / \mathrm{L}$ agarose gel electrophoresis.

\section{Availability Test of RHDV2 Template Fragment}

To assess the feasibility of the target RHDV2 gene fragment in pMD-19T-RHDV2 for RHDV2 RT-LAMP detection The RNA extraction of RHDV and RT reaction were subsequently performed by using the RNAiso Plus reagent (BK1501) and PrimeScriptTM RT Reagent kit (BK1901).Using P3/P4 as primers and $2 \mu \mathrm{L}$ of the RT reaction products as templates, the corresponding RHDV VP60 target fragments were amplified by PCR(the PCR reaction system and procedure were both same as above), and then were separated and sequenced as described in[13].

\section{E. Basic RT-LAMP Reaction}

The RT-LAMP reaction mixture $(25 \mu \mathrm{L})$ contained $2.5 \mu \mathrm{L}$ of $10 \times$ ThermoPol buffer, $1 \mu \mathrm{L}$ ( 8 units) of Bst 2.0 DNA polymerase, $1.5 \mu \mathrm{L}$ of $\mathrm{MgSO}_{4}(100 \mathrm{mM}), 3 \mu \mathrm{L}$ of betaine $(8 \mathrm{mM}), 6 \mu \mathrm{L}$ of dNTP $(2.5 \mathrm{mM}), 1 \mu \mathrm{L}$ of each primers $(\mathrm{R} 2 \mathrm{~F} 3$ $(5 \mu \mathrm{M})$, R2B3 $(5 \mu \mathrm{M})$, R2FIP $(50 \mu \mathrm{M})$ and R2BIP $(50 \mu \mathrm{M}))$,

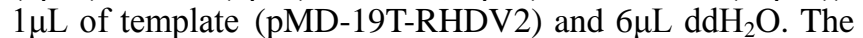
reaction mixture was incubated at $64{ }^{\circ} \mathrm{C}$ for $2 \mathrm{~h}$, then heated at $85{ }^{\circ} \mathrm{C}$ for $2 \mathrm{~min}$ in a laboratory water bath (DSY21-8, China), and the amplified products were analyzed in $15 \mathrm{~g} / \mathrm{L}$ agarose gel in TAE buffer. The results were also visualized directly with the naked eye according to the white precipitate of magnesium pyrophosphate generated in the reaction or the green color changed with $254 \mathrm{~nm}$ UV light by the addition of diluted SYBR GreenI. To confirm the specificity of amplification products, $20 \mu \mathrm{L}$ of the reaction mixture which containing $3 \mu \mathrm{L}$ of the LAMP products was digested with EcoR II at $37{ }^{\circ} \mathrm{C}$ for $2 \mathrm{~h}$.

\section{F. Optimization of RHDV2 RT-LAMP Reaction Conditions}

In the optimum conditions assay, the RT-LAMP reaction mixture as described above were used, and the reaction conditions were determined to be as follows: a gradient of incubation degree from $59^{\circ} \mathrm{C}$ to $66{ }^{\circ} \mathrm{C}$ for $90 \mathrm{~min}$, and at the determined incubation degree for $30 \mathrm{~min}, 60 \mathrm{~min}, 90 \mathrm{~min}$ and $2 \mathrm{~h}$, respectively. In all, $5 \mu \mathrm{L}$ of RT-LAMP products were analyzed using $15 \mathrm{~g} / \mathrm{L}$ agarose gel electrophoresis.

\section{G. Sensitivity Test of RT-LAMP}

Using the recombinant plasmids constructed above as standard control samples, the pMD-19T-RHDV2, which concentration were determined by a ND-1000 ultraviolet spectrophotometer (Nano Drop Co., Ltd, USA), were diluted in a 10-fold serial, and each dilution was tested by the RT-LAMP using the determined reaction conditions. The number of target gene fragment molecule copies in each reaction was calculated according to the formula in [12], then the sensitivity of RT-LAMP was assessed.

\section{H. Specificity Test of RT-LAMP}

The specificity tests of RHDV2 RT-LAMP was carried out through comparing the pMD-19T-RHDV2 to RHDV(RHDV-Sch01), EBHSV(pGM-T-EBHSV), Pasteurella multocida, Salmonellaand E.coli etc common rabbit pathogens. RNA and DNA (cDNA) templates were prepared using a commonly reported method from [13].

\section{Primary Application Test}

5 RHDV experimentally samples infected with the RHDV-Sch01 and 25 clinical samples were detected by this RT-LAMP method using the reaction conditions determined above.

\section{RESULT}

\section{A. Identification and Availability Test of RHDV2 Target VP60 Gene Fragment}

To develop the RHDV2 RT-LAMP detection method, the constructed pMD-19T-RHDV2 was identified by PCR (using P1/P2 and MR13-47/RV-M as primers separately) and sequencing analysis, and then the availability for RT-LAMP detection was assessed as above in the method section. The results showed a DNA band about 435 bp in the length (see Fig. 1A, lane2)and a DNA band longer than 435 bp (see Fig. 1A, lane1)was amplified by PCRs, and the sequencing results showed the RHDV2 VP60 target DNA fragment had a $100 \%$ homology with the reference sequences(KP129339). In the availability test, the corresponding RHDV VP60 target fragment about $441 \mathrm{bp}$, which had a 100\% homology with the reference sequences(KF537692) in GenBank, was successfully amplified by RT-PCR method (see Fig. 1B, lane5). The result suggests that it is reliable to use the RHDV2 VP60 DNA fragment about $435 \mathrm{bp}$ which corresponding to the RHDV VP60 target fragment about 441 bp as target gene for RT-LAMP study. 


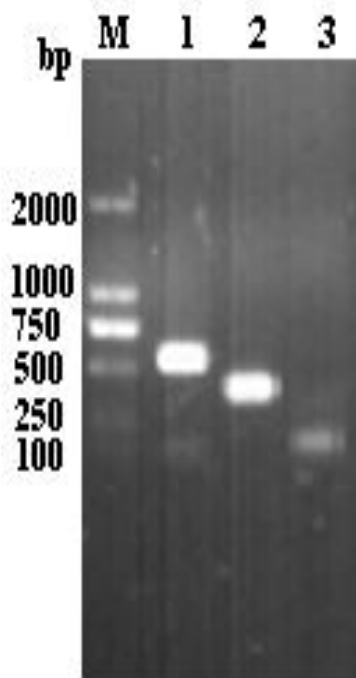

A

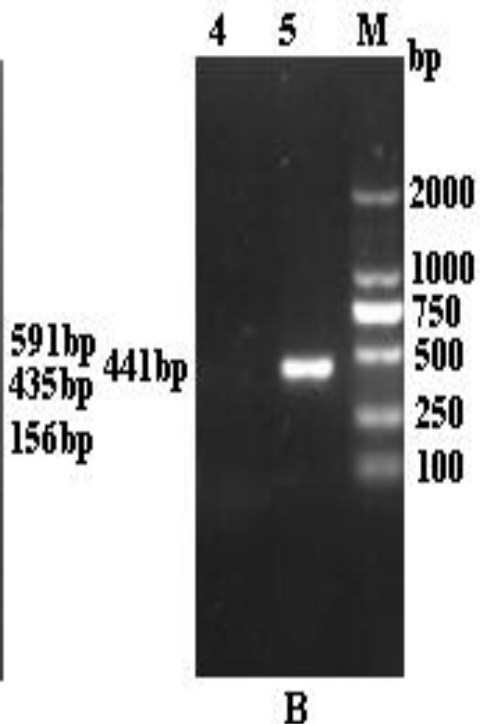

B

Figure 1. Amplification results of target DNA fragments A:Identification of pMD-19T-RHDV2 B: RT-PCR results of RHDV M: DNA marker DL2000; 1: pMD-19T-RHDV2 PCR products using primers MR13-47/RV-M;2: pMD-19T-RHDV2 PCR products using primers P1/P2; pMD-19T vector PCR products using primers MR13-47/RV-M; 4:Negative control; 5: RT-PCR products of corresponding RHDV VP60 fragment

\section{B. Basic RT-LAMP Reaction}

The RHDV basic RT-LAMP was carried out using pMD-19T-RHDV2 as template and incubated at $64{ }^{\circ} \mathrm{C}$ for 2 $\mathrm{h}$, then the amplification products were analyzed by using agarose gel electrophoresis, enzyme- digestion and visual inspection as described in the method section above. The results (as shown in Fig.2) showed the pMD-19T-RHDV2 was amplified at $64{ }^{\circ} \mathrm{C}$ with a ladder-like pattern bands from about 119 bp on the gel (Fig.2(A) lane1), which specificity were conformed by the smaller digestion products from 100 bp to 119 bp with EcoRII (Fig.2(A) lane3). The positive reaction could also be visualised clearly by the white precipitate of magnesium pyrophosphate (Fig.2(B) tube4) and the green color produced after the addition of diluted SYBR GreenI (Fig.2(C) tube6), while the pMD-19T vector template control reactions had no amplifications and visual inspection changes ( Fig.2 lane2, tube5 and 7).

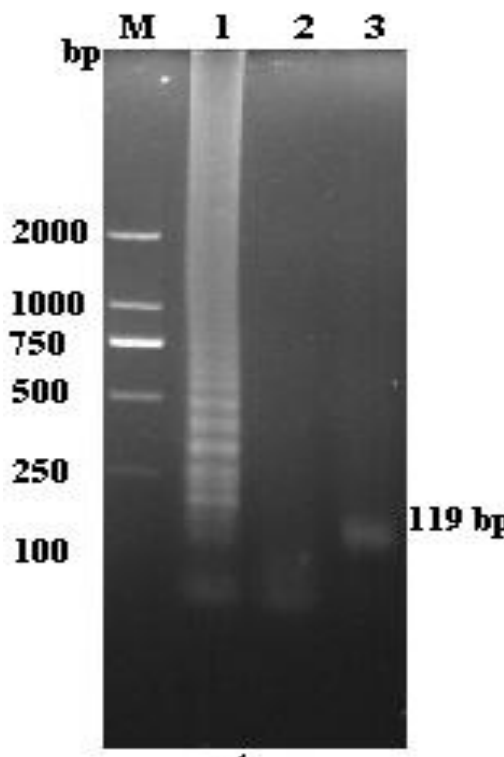

A

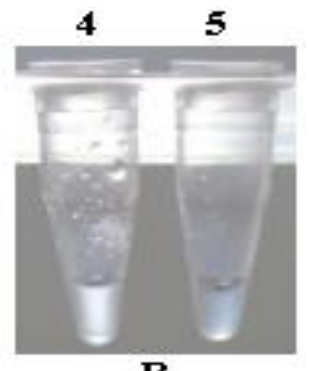

B

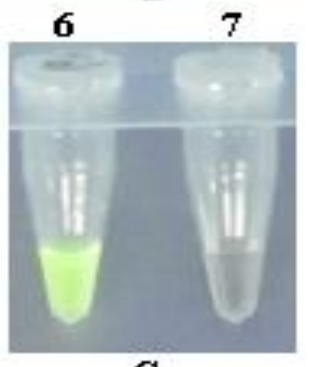

C

Figure 2. Basic RT-LAMP results of RHDV2

A:Agarose gel electrophoresis; B:Visual inspec- tion by turbidity; C:visual inspection by adding SYBR GreenI; M: DNA marker DL2000;1,4,6: RT-LAMP reaction products of pMD-19T-RHDV2; 2,5,7: Negative control (pMD-19T vector); 3:The RT-LAMP positive products digested with EcoR II

\section{Optimization of RHDV2 RT-LAMP Reaction Conditions}

The RT-LAMP reaction conditions were determined based on the optimization of reaction temperatures and reaction time as above. The results showed that that lane4, lane5, lane6, lane7, lane8 and lane11, lane12 had clearer ladder-like reaction bands (as shown in Fig.3). Then the optimal temperature and reaction times for the RT-LAMP were selected at $63.5^{\circ} \mathrm{C}$ for $90 \mathrm{~min}$ for the specificity and amplification efficiency, and the $25 \mu \mathrm{L}$ reaction volume as described in the method section above was determined, and the optimum RT reaction volume components and conditions were the same as the instructions of Primerscript RT reagent Kit according to the availability test results. 


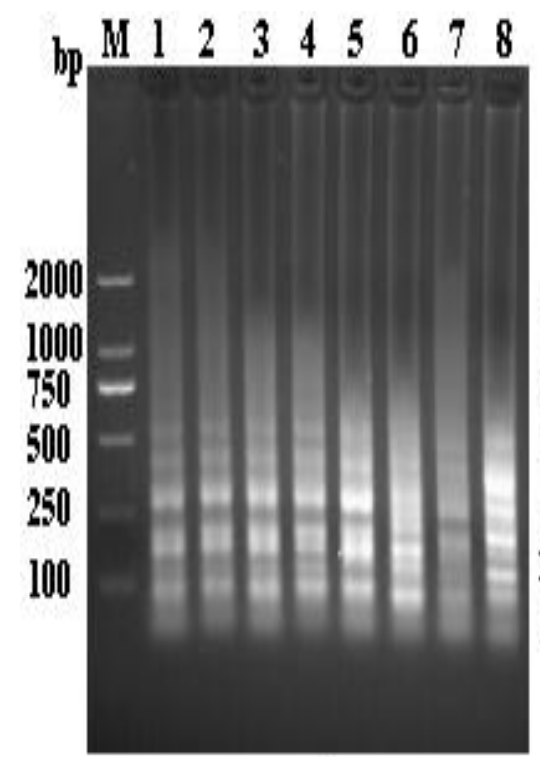

A

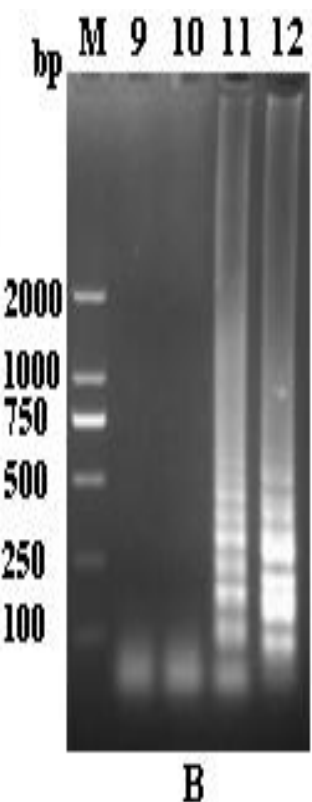

B
Figure 3. The results of reaction conditions optimization. A: The optimization test result of reaction temperatures; $\mathrm{B}$ : The optimization test result of reaction time; $1: 59{ }^{\circ} \mathrm{C} ; 2: 60{ }^{\circ} \mathrm{C} ; 3: 60.8{ }^{\circ} \mathrm{C} ; 4: 61.8$ ${ }^{\circ} \mathrm{C} ; 5: 62.9{ }^{\circ} \mathrm{C} ; 6: 64{ }^{\circ} \mathrm{C} ; 7: 64.8{ }^{\circ} \mathrm{C} ; 8: 66{ }^{\circ} \mathrm{C} ; 9: 30 \mathrm{~min} ; 10: 60 \mathrm{~min} ; 11: 90 \mathrm{~min}$; $12: 2 \mathrm{~h}$

\section{Sensitivity and Specificity of RHDV2 RT-LAMP}

In the sensitivity test, the pMD-19T-RHDV2 diluted at different concentrations were tested, The results(as shown in Fig.4) showed the ladder-like pattern from about 119bp could be observed in lane 1 to lane5(180copies $/ \mu \mathrm{L})$, indicating that the LAMP detection limit was 180 copies $/ \mu \mathrm{L}$ of the target gene fragments. For the specificity,There was a clear expected ladder-like pattern of electrophoretic bands observed only for the pMD-19T-RHDV2 detection (see Fig.5,lane1), and no specific amplification for the detection of RHDV(RHDV-Sch01), EBHSV(pGM-T-EBHSV), Pasteurella multocida, E.coli and Salmonella etc common rabbit pathogens.(see Fig.5 lane2-7).

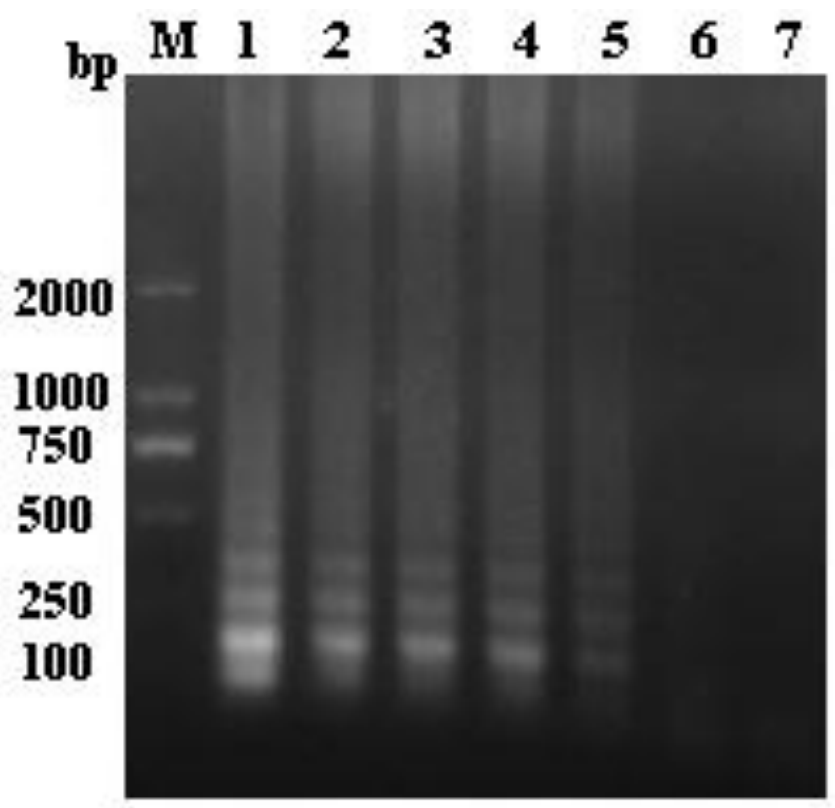

Figure4 The sensitivity assay results of RHDV2 RT-LAMP $1: 1.8 \times 10^{6} \mathrm{copies} / \mu \mathrm{L} ; 2: 1.8 \times 10^{5} \mathrm{copies} / \mu \mathrm{L} ; 3: 1.8 \times 10^{4}$ copies $/ \mu \mathrm{L}$; 4:1.8×10 ${ }^{3}$ copies $/ \mu \mathrm{L} ; 5: 180$ copies $/ \mu \mathrm{L} ; 6: 18$ copies $/ \mu \mathrm{L} ; 7: 1.8$ copies $/ \mu \mathrm{L}$

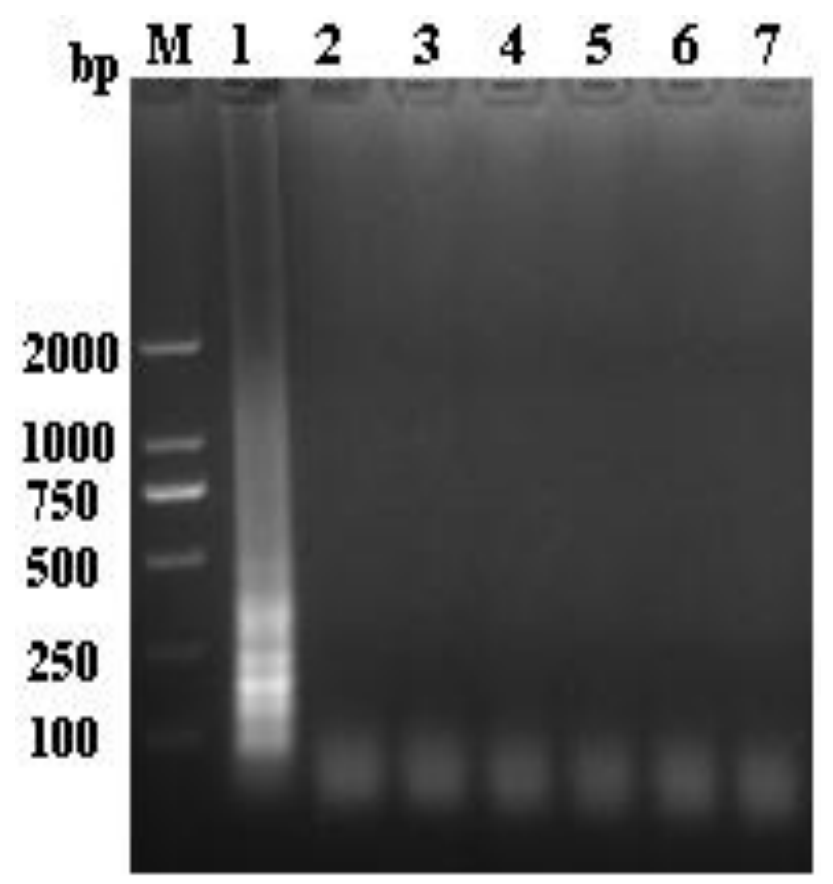

Figure 5. The specificity assay results of RHDV2 RT-LAMP 1: pMD-19T-RHDV2; 2: NTC (ddH2O); 3: RHDV(RHDV-Sch01); 4: EBHSV(pGM-T-EBHSV); 5: Pasteurella multocida; 6: E.coli; 7:

$$
\text { Salmonella }
$$

\section{E. Primary Application of RHDV2 RT-LAMP}

5 RHDV experimentally RHDV samples and 25 clinical samples were detected by this method, all the detection results were negative, this results may indicate there are no RHDV2 in the detected rabbits until now. 


\section{DISCUSSION AND CONCLUSIONS}

Since RHDV was reported in 1984, it has caused important economic losses in many countries and was also regard as a significant negative ecological impact among wild rabbit populations indirectly on its dependant predators $[3,4]$. Interestingly, RHDV had been considered as an agent for rabbit control and was legally or illegally introduced to Australia and New Zealand in 1990s, as the rabbit was an important agricultural pest and a major threat to the endemic wildlife flora and fauna [4, 14, 15]. Many studied showed that RHDV2, as an member of genus Lagovirus of the family Caliciviridae, was different with the classical RHDV and RHDVa strains, and it could affect younger rabbits, RHD-vaccinated rabbits and some hare species. The molecular epidemiology of RHDV2 had revealed that it was rapidly replacing the previously circulating classical strains in France, Spain and Portugal[16,17].Therefore, RHDV2 may has the more serious threat to the developing of rabbit-breeding and the ecosystem balance of the rabbit centered .

To our knowledge, there is no RHDV2 infection in China, So this RT-LAMP method was developed through identification of target gene fragments, availability test, optimization of reaction conditions, sensitivity and specificity tests, and the application tests based on the pMD-19T-RHDV2 containing the conserved gene fragments about 435 bp of RHDV2VP60 constructed by [13], and this is the first research reported on the RHDV2 RT-LAMP method study.

In conclusion, the RT-LAMP assays exhibits good sensitivity and specificity repeatability. Its detection limit could reach 180 copies $/ \mu \mathrm{L}$ of the target gene segments, and there were no amplification for the EBHSV (pGM-T-EBHSV), RHDV (RHDV-Sch01), E.coli, Pasteurella multocida and Salmonella etc common pathogens detection by the RT-LAMP method. This study supplies a new useful technology in reserve for RHDV2 detection to help prevent and control RHDV2 in China.

\section{ACKNOWLEDGEMENT}

This study was supported by Grants from the National Natural Science Foundation of China (31402222), the planning subject of 'the twelfth five-year-plan' in national science and technology for the rural development in China (2013BAD12B04).Liu Yadong, Zhou lijun, Zhang Han, Xiang Xiaoxue, Luo yilin, Li Yan and Meng Zhengqun should also be considered as first authors, Corresponding author: Wang Yin, Yang Zexiao.

\section{REFERENCES}

[1] G. Le Gall, C.Arnauld, E.Boilletot, J.P.Morisse and D.Rasschaert, "Molecular epidemiology of rabbit haemorrhagic disease virus outbreaks in France during 1988 to 1995," J Gen Virol, vol. 79, Jan 1998, pp. 11-16, doi: 10.1099/0022-1317-79-1-11.

[2] B Gromadzka, B Szewczyk, G Konopa, A Fitzner and A Kesy, "Recombinant VP60 in the form of virion-like particles as a potential vaccine against rabbit hemorrhagic disease virus," Acta Biochim Pol. vol. 53, May 2006, pp. 371-376.

[3] F Chen. Veterinary Lemology, the 5th ed Beijing:China Agriculture Press,Apr.2008,pp.410-412.

[4] J Abrantes, W van der Loo, J Le Pendu and PJ Esteves, "Rabbit haemorrhagic disease (RHD) and rabbit haemorrhagic disease virus (RHDV): a review," Veterinary Research, vol. 43, Feb 2012, pp.10-19, doi: 10.1186/1297-9716-43-12

[5] L Yang, F Wang, B Hu, J Xue, Y Hu, B Zhou, D Wang and W Xu, "Development of an RT-PCR for rabbit hemorrhagic disease virus (RHDV) and the epidemiology of RHDV in three eastern provinces of China," J Virol Methods,vol. 151, Jul 2008 ,pp.24-29, doi:10.1016/j.jviromet.2008.04.003

[6] G Le Gall-Reculé, A Lavazza, S Marchandeau, S Bertagnoli, F Zwingelstein, P Cavadini, N Martinelli, G Lombardi, J L Guérin, E Lemaitre, A Decors, S Boucher, B Le Normand and L Capucci, "Emergence of a new lagovirus related to Rabbit Haemorrhagic Disease Virus," Veterinary Research, vol. 44, Sep 2013,pp.81, doi:10.1186/1297-9716-44-81

[7] J Bárcena, B Guerra, I Angulo, J González, F Valcárcel, C.P. Mata, J.R. Castón, E .Blanco and A .Alejo, "Comparative analysis of rabbit hemorrhagic disease virus (RHDV) and new RHDV2 virus antigenicity, using specific virus-like particles," Vet Res, vol. 46, Sep 2015,pp.106. doi: 10.1186/s13567-015-0245-5.

[8] A Martin-Alonso, N Martin-Carrillo, K Garcia-Livia, B Valladares and $\mathrm{P}$ Foronda, "Emerging rabbit haemorrhagic disease virus 2 (RHDV2) at the gates of the African continent," Infect Genet Evol, vol. 44,Oct 2016,pp.46-50. doi: 10.1016/j.meegid.2016.06.034.

[9] T Notomi, H Okayama, H Masubuchi, T Yonekawa, K Watanabe, NAmino and T Hase, "Loop-mediated isothermal amplification of DNA," Nucleic Acids Research, vol. 28, Jun 2000,pp. E63

[10] Z Yang, Y Hou, H Zeng, X Yao, Y Wang, H Liu, B Liu, X Wu and B Peng. "Development and Application of LAMP Method for Porcine Circovirus type2 Detection," Chinese Veterinary Science, vol.44, Feb 2014, pp.152-158.

[11] Z Yang, H Zeng, Y Wang, X Yao and K Wang, "Establishment of a RT-LAMP Method for the Rabbit Hemorrhagic Disease Virus Detection," Biotechnology:An Indian Journal, vol.8,Feb 2013,pp. 164-269.

[12] Z Yang, G Li, Y Hou, X Yao, R Ren, H Ya, S Peng, X Lin and YWang, "Artificial Synthesis of Conserved Segment $\mathrm{S}$ Gene Fragment of Rift Valley Fever Virus and Preliminary Study of Its Reverse Transcription Loop-Mediated Isothermal Amplification Detection Method," Tropical Journal of Pharmaceutical Research, vol. 14,Dec 2015,pp. 2193-2200.

[13] Z Yang, X Zhao, Y Li, B Wang, X Yao, Y Wang, Y Geng, Z Meng and Y Bai, "Artificial synthesis of VP60 conserved Gene fragment of rabbit hemorrhagic disease virus type 2 and preliminary study of its RT-PCR detection method," Acta Agriculturae Zhejiangensis, vol. 28, Oct 2016,pp.1650-1656.

[14] J A Gibb and J M Williams, The rabbit in New Zealand. In The European rabbit: the history and biology of a successful colonizer. Oxford: Oxford University Press, 1994, pp.158-200.

[15] F Fenner, "Deliberate introduction of the European rabbit, Oryctolagus cuniculus, into Australia," Revue scientifique et technique, vol. 29, Apr 2010,pp.103-111.

[16] K.P. Dalton, I.Nicieza, J.Abrantes, P.J.Esteves and F.Parra, "Spread of new variant RHDV in domestic rabbits on the Iberian Peninsula,"Veterinary Microbiology, vol. 169, Feb 2014, pp. 67-73, doi: 10.1016/j.vetmic.2013.12.015.

[17] AM Lopes, J Correia, J Abrantes, P Melo, M Ramada, MJ Magalhães, PC Alves and PJ Esteves, "Is the new variant RHDV replacing genogroup 1 in Portuguese wild rabbit populations?" Viruses, vol. 7, Dec 2014, pp.27-36, doi: 10.3390/v7010027. 